Скопје, Македонија

\title{
SOME RESULTS CONCERNING THE ANALYTIC REPRESENTATION OF CONVOLUTION
}

\author{
VASKO RECKOVSKI, EGZONA ISENI, AND VESNA MANOVA ERAKOVIKJ
}

\begin{abstract}
In this paper we will prove some results concerning the analytic representation of the convolution of some functions and distributions.
\end{abstract}

\section{INTRODUCTION}

We use the standard notation from the Schwartz distribution theory.

The boundary value representation has been studied for a long time and from different points of view.

One of the first result is that if $f \in L^{1}$, then the function

$\hat{f}(z)=\frac{1}{2 \pi i}<f(t), \frac{1}{t-z}>$, for $\operatorname{Im} z \neq 0$ is the Cauchy representation of $f$ i.e.

$\lim _{y \rightarrow 0^{+}}<\hat{f}(x+i y)-\hat{f}(x-i y), \varphi(x)>=<f, \varphi>$, for every $\varphi \in D$.

$D_{L^{p}}, 1 \leq p<\infty$ denotes the space of all infinitely differentiable functions $\varphi$ for which $\varphi^{(\beta)} \in L^{p}$ for each n-tuple $\beta$ of nonnegative integers.

$B=D_{L^{\infty}}$ is the space of all infinitely differentiable functions which are bounded on $\mathbb{R}^{n}$.

$\dot{B}$ is the subspace of $B$ that consists of all functions $\varphi \in B$ which vanish at infinity together with each of their derivatives.

The topology of $D_{L^{p}}$ is given in terms of the norms

$$
\|\varphi\|_{m, p}=\left(\int_{\mathbb{R}^{n}}\left|\varphi^{(\beta)}(x)\right|^{p} d x\right)^{1 / p},|\beta| \leq m, \quad m=0,1,2,3, \ldots .
$$

A sequence of functions $\left(\varphi_{\lambda}\right)$ of $D_{L^{p}}$ converges to a function $\varphi$ in the topology of $D_{L^{p}}, 1 \leq p<\infty$ as $\lambda \rightarrow \lambda_{0}$ if each $\varphi_{\lambda} \in D_{L^{p}}, \varphi \in D_{L^{p}}$, and

$$
\lim _{\lambda \rightarrow \lambda_{0}}\left\|\varphi_{\lambda}^{(\beta)}-\varphi^{(\beta)}\right\|_{L^{p}}=\lim _{\lambda \rightarrow \lambda_{0}}\left(\int_{\mathbb{R}^{n}}\left|\varphi_{\lambda}^{(\beta)}(x)-\varphi^{(\beta)}(x)\right|^{p} d x\right)^{1 / p}=0, \text { for }
$$
every $\beta$.

A sequence of functions $\left(\varphi_{\lambda}\right)$ converges to the function $\varphi$ in $\dot{B}$ as $\lambda \rightarrow \lambda_{0}$ if each $\varphi_{\lambda} \in \dot{B}, \varphi \in \dot{B}$, and

2000 Mathematics Subject Classification. Primary 46F20; Secondary 44A15, 46F12.

Key words and phrases. convolution, distribution, analytic representation. 


$$
\lim _{\lambda \rightarrow \lambda_{0}}\left\|\varphi_{\lambda}^{(\beta)}-\varphi^{(\beta)}\right\|_{L^{\infty}}=0
$$

$D^{\prime}{ }_{L^{p}}, 1 \leq p<\infty$ is the space of all continuous linear functional on $D_{L^{q}}$, where $\frac{1}{p}+\frac{1}{q}=1 . D_{L^{1}}^{\prime}$ is the space of all continuous linear functional on $\dot{B}$.

The following theorem gives the structures of $D^{\prime}{ }_{L^{p}}$.

Structure Theorem. A distribution $\Lambda$ belongs to $D^{\prime}{ }_{L^{p}}, 1 \leq p<\infty$ if and only if $\Lambda$ is a finite sum of distributional derivatives of functions in $L^{p}$, i.e. there is an integer $m \geq 0$ depending only on $\Lambda$ such that

$\Lambda=\sum_{|\beta| \leq m} f_{\beta}{ }^{(\beta)}$, where $f_{\beta} \in L^{p}$ for each $\beta,|\beta| \leq m$.

If $f, g \in L^{1}$ then $\int_{\mathbb{R}}|f(x-y) g(y)| d y<\infty$ for almost all $x$.

The function $h(x)=(g * f)(x)=\int_{\mathbb{R}} f(x-y) g(y) d y$ belongs to $L^{1}(\mathbb{R})$, $\|h\|_{1} \leq\|f\|_{1}\|g\|_{1}$ and $g * f=f * g . h$ is called the convolution of $f$ and $g$.

If $f \in L^{1}(\mathbb{R}), g \in L^{p}(\mathbb{R})$ for $1 \leq p<\infty$ then for almost all $x \in \mathbb{R}^{1}$, the functions of $y, f(x-y) g(y)$ and $f(y) g(x-y)$ are in $L^{1}(\mathbb{R})$. For all such $x$, and $(g * f)(x)=\int_{\mathbb{R}} f(x-y) g(y) d y$ we have $f * g=g * f$ a.e., $f * g \in L^{p}$ and $\|f * g\|_{p} \leq\|f\|_{1}\|g\|_{p}$.

The proof that $h=f * g$ belongs to $L^{p}$ for $1 \leq p<\infty$ is given in [5].

The following theorem is also known.

Theorem. Let $f$ and $g$ be in $L^{1}$ and let $h=g * f=f * g$. Then $h$ has Cauchy representation

$$
\hat{h}(z)=\frac{1}{2 \pi i} \int \frac{h(t)}{t-z} d t=\int_{\mathbb{R}} f(t) \hat{g}(z-t) d t=\int_{\mathbb{R}} g(t) \hat{f}(z-t) d t, z=x+
$$

$i y, \operatorname{Im} z \neq 0$.

\section{MAIN RESULTS}

We will prove some results concerning the analytic representation of the convolution $h=f * g$ for $f \in L^{1}, g \in L^{p}$.

Theorem 1. Let $f \in L^{1}(\mathbb{R})$ and $g \in L^{p}(\mathbb{R}), 1 \leq p<\infty$ and let $h=$ $f * g$. Then $h$ has the Cauchy representation $\hat{h}(z)=\frac{1}{2 \pi i} \int \frac{h(t)}{t-z} d t, z=x+$ $i y, \operatorname{Im} z \neq 0$.

Proof. For $\varphi \in D$,

$$
\begin{aligned}
& \lim _{y \rightarrow 0^{+}} \int_{\mathbb{R}}[\hat{h}(x+i y)-\hat{h}(x-i y] \varphi(x) d x= \\
& \lim _{y \rightarrow 0^{+}} \int_{\mathbb{R}} \frac{1}{2 \pi i}\left[\int_{\mathbb{R}}\left(\frac{h(t)}{t-z}-\frac{h(t)}{t-\bar{z}}\right) d t\right] \varphi(x) d x=
\end{aligned}
$$




$$
\lim _{y \rightarrow 0^{+}} \int_{\mathbb{R}} \frac{1}{2 \pi i}\left(\int_{\mathbb{R}} \int_{\mathbb{R}}\left[\frac{f(u) g(t-u) d u}{t-z}-\frac{f(u) g(t-u) d u}{t-\bar{z}}\right] d t\right) \varphi(x) d x .
$$

The above integrals exist by the Hölder inequality, hence applying Fubini's theorem, we may change the order of integration and get that

$$
\begin{aligned}
& \lim _{y \rightarrow 0^{+}} \int_{\mathbb{R}}[\hat{h}(x+i y)-\hat{h}(x-i y] \varphi(x) d x= \\
& \lim _{y \rightarrow 0^{+}} \frac{1}{2 \pi i} \int_{\mathbb{R}}\left(\frac{\varphi(x)}{t-z}-\frac{\varphi(x)}{t-\bar{z}}\right) d x \int_{\mathbb{R}} f(u) d u \int_{\mathbb{R}} g(t-u) d t= \\
& \lim _{y \rightarrow 0^{+}} \frac{y}{\pi} \int_{\mathbb{R}} \frac{\varphi(x)}{|t-z|^{2}} d x \int_{\mathbb{R}} f(u) d u \int_{\mathbb{R}} g(t-u) d t .
\end{aligned}
$$

Now by the Lemma $5.4[1]$, we get that $\frac{y}{\pi} \int_{\mathbb{R}} \frac{\varphi(x)}{|t-z|^{2}} d x=\hat{\varphi}(t+i y)$ and that $\int_{\mathbb{R}} f(u) d u \int_{\mathbb{R}} g(t-u) \hat{\varphi}(t+i y) d t$ converges to $\int_{\mathbb{R}} f(u) d u \int_{\mathbb{R}} g(t-u) \varphi(t) d t$ as $y \rightarrow 0^{+}$.

Finally, with one more use of Fubini's theorem, we get

$$
\begin{aligned}
& \lim _{y \rightarrow 0^{+}} \int_{\mathbb{R}}[\hat{h}(x+i y)-\hat{h}(x-i y] \varphi(x) d x= \\
& \int_{\mathbb{R}} f(u) g(t-u) d u \int_{\mathbb{R}} \varphi(t) d t=\int_{\mathbb{R}}(f * g)(t) \varphi(t) d t=<f * g, \varphi>.
\end{aligned}
$$

Theorem 2. Suppose that the sequence $\left\{f_{n}\right\}$ converges to $f$ in $L^{1}$ sense and $g \in L^{1}$. Let $h_{n}=f_{n} * g$. Then the sequence $\left\{h_{n}\right\}$ converges to $h=f * g$ in $L^{1}$. If $\hat{h_{n}}(z)$ is analytic representation of every $h_{n}$ for $n=1,2,3, \ldots$ then the sequence $\left\{\hat{h_{n}}(z)\right\}$ converges uniformly to $\hat{h}(z)$ on compact subsets of $\mathbb{C} / \mathbb{R}$ and $\hat{h}(z)$ is analytic representation of $h$.

Proof. Let us consider the difference

$$
\left|\int_{\mathbb{R}} h_{n}(x) d x-\int_{\mathbb{R}} h(x) d x\right|=\left|\int_{\mathbb{R}}\left(f_{n} * g\right)(x) d x-\int_{\mathbb{R}}(f * g)(x) d x\right|
$$




$$
\begin{aligned}
& =\left|\int_{\mathbb{R}} \int_{\mathbb{R}}\left[f_{n}(y) g(x-y) d y-f(y) g(x-y) d y\right] d x\right| \\
& =\left|\int_{\mathbb{R}} \int_{\mathbb{R}}\left[f_{n}(y)-f(y)\right] g(x-y) d y d x\right|
\end{aligned}
$$

By Fubini's theorem, in the last integral, we may change the order of integration and get that

$$
\left|\int_{\mathbb{R}} h_{n}(x) d x-\int_{\mathbb{R}} h(x) d x\right| \leq \int_{\mathbb{R}}\left|f_{n}(y)-f(y)\right| d y \int_{\mathbb{R}}|g(x-y)| d x .
$$

Since $f_{n} \rightarrow f$ in $L^{1}$ and by Lebesgue's dominated convergence theorem we get that $h_{n} \rightarrow h$ in $L^{1}$. The facts that the sequence $\left\{\hat{h_{n}}(z)\right\}$ converges uniformly to $\hat{h}(z)$ on compact subsets of $\mathbb{C} / \mathbb{R}$ and that $\hat{h}(z)$ is analytic representation of $h$ follow from the Theorem 2.1 in [4] and Theorem 1 in this paper.

Theorem 3. Suppose that $f \in L^{1}$ and the sequence $\left\{g_{n}\right\}, g_{n} \in L^{p}$ for $1 \leq p<\infty$ is such that $g_{n} \rightarrow g$ in $L^{p}$. Then the sequence $\left\{h_{n}\right\}$, $h_{n}=f * g_{n}$ converges to $h$ in $L^{p}$ for $1 \leq p<\infty$ and $h=f * g$. If $\hat{h_{n}}(z)$ is analytic representation of $h_{n}$ for $n=1,2,3, \ldots$ respectively, then the sequence $\left\{\hat{h_{n}}(z)\right\}$ converges uniformly to $\hat{h}(z)$ on compact subsets of $\mathbb{C} / \mathbb{R}$ and $\hat{h}(z)$ is analytic representation of $h$.

Proof. Let $h_{n}=f * g_{n}$ and $h=f * g$. Then

$$
\begin{aligned}
& \left|\int_{\mathbb{R}} h_{n}(x) d x-\int_{\mathbb{R}} h(x) d x\right|=\left|\int_{\mathbb{R}} \int_{\mathbb{R}}\left[f(y) g_{n}(x-y) d y-f(y) g(x-y)\right] d y d x\right| \\
& \leq \int_{\mathbb{R}}\left|\int_{\mathbb{R}}\left[f(y) g_{n}(x-y)-f(y) g(x-y)\right] d y\right| d x
\end{aligned}
$$

Applying Fubini's theorem in the last integral, we have that $\left|\int_{\mathbb{R}} h_{n}(x) d x-\int_{\mathbb{R}} h(x) d x\right| \leq \int_{\mathbb{R}}|f(y)| d y \int_{\mathbb{R}}\left|g_{n}(x-y)-g(x-y)\right| d x$.

Now we may apply the Lebesgue dominated convergence theorem and we get that $h_{n} \rightarrow h$ in $L^{p}$ sense. The facts that the sequence $\left\{\hat{h_{n}}(z)\right\}$ 
converges uniformly to $\hat{h}(z)$ on compact subsets of $\mathbb{C} / \mathbb{R}$ and that $\hat{h}(z)$ is analytic representation of $h$ follow from the Theorem 2.1 in [4] and Theorem 1 in this paper.

We denote by $L_{Q}^{p}=\left\{g \mid g\right.$ is a measurable function on $\mathbb{R}$ and $\left.\frac{g}{Q} \in L^{p}\right\}$, where $\mathrm{Q}$ is a function without real roots.

Theorem 4. Suppose that $f \in L^{1}(\mathbb{R}), \mathrm{Q}$ is a function without real roots and $g$ is measurable function on $\mathbb{R}$ that belongs to the space $L_{Q}^{p}$. The convolution of the functions $f \in L^{1}$ and $\frac{g}{Q} \in L^{p}, h=f *\left(\frac{g}{Q}\right)$ is such that $h \in L^{p},\|h\|_{p} \leq\|f\|_{1}\left\|\frac{g}{Q}\right\|_{p}$ and $h$ has Cauchy representation $\hat{h}(z)=\frac{1}{2 \pi i}<h, \frac{1}{t-z}>$.

Proof. The fact that $h \in L^{p},\|h\|_{p} \leq\|f\|_{1}\left\|\frac{g}{Q}\right\|_{p}$ can be easily proven as in the introduction part.

Let $\varphi \in D$ be arbitrary function. Then we have

$$
\begin{aligned}
& \lim _{\varepsilon \rightarrow 0^{+}} \int_{\mathbb{R}}[\hat{h}(x+i \varepsilon)-\hat{h}(x-i \varepsilon] \varphi(x) d x= \\
& =\lim _{\varepsilon \rightarrow 0^{+}} \int_{\mathbb{R}}\left[\frac{1}{2 \pi i} \int_{\mathbb{R}}\left(\frac{h(t)}{t-z}-\frac{h(t)}{t-\bar{z}}\right) d t\right] \varphi(x) d x= \\
& =\lim _{\varepsilon \rightarrow 0^{+}} \int_{\mathbb{R}}\left[\frac{1}{2 \pi i} \int_{\mathbb{R}} \frac{d t}{t-z} \int_{\mathbb{R}} f(u) \frac{g(u-t)}{Q(u-t)} d u-\right. \\
& \left.-\frac{1}{2 \pi i} \int_{\mathbb{R}} \frac{d t}{t-\bar{z}} \int_{\mathbb{R}} f(u) \frac{g(u-t)}{Q(u-t)} d u\right] \varphi(x) d x .
\end{aligned}
$$

Since the integrals exist, by Fubini's theorem, we may change the order of integration and get

$$
\begin{aligned}
& \lim _{\varepsilon \rightarrow 0^{+}} \int_{\mathbb{R}}[\hat{h}(x+i \varepsilon)-\hat{h}(x-i \varepsilon] \varphi(x) d x= \\
& \lim _{\varepsilon \rightarrow 0^{+}} \int_{\mathbb{R}} \varphi(x) d x \frac{y}{\pi} \int_{\mathbb{R}} \frac{d t}{|t-z|^{2}} \int_{\mathbb{R}} f(u) \frac{g(u-t)}{Q(u-t)} d u= \\
& \lim _{\varepsilon \rightarrow 0^{+}} \frac{y}{\pi} \int_{\mathbb{R}} \frac{\varphi(x) d x}{|t-z|^{2}} \int_{\mathbb{R}} f(u) \int_{\mathbb{R}} \frac{g(u-t)}{Q(u-t)} d u d t=
\end{aligned}
$$




$$
\lim _{\varepsilon \rightarrow 0^{+}} \frac{y}{\pi} \int_{\mathbb{R}} \frac{\varphi(x) d x}{|t-z|^{2}} \int_{\mathbb{R}} f(u) \frac{g(u-t)}{Q(u-t)} d u d t .
$$

By the Lebesgue dominated convergence theorem and the Lemma 5.4 in [1], we have that

$$
\lim _{\varepsilon \rightarrow 0^{+}} \int_{\mathbb{R}}\left[\hat{h}(x+i \varepsilon)-\hat{h}(x-i \varepsilon] \varphi(x) d x=\int_{\mathbb{R}} f(u) d u \int_{\mathbb{R}} \frac{g(u-t)}{Q(u-t)} \varphi(t) d t . .\right.
$$

One more application of Fubini's theorem gives that

$$
\begin{aligned}
& \lim _{\varepsilon \rightarrow 0^{+}} \int_{\mathbb{R}}[\hat{h}(x+i \varepsilon)-\hat{h}(x-i \varepsilon] \varphi(x) d x= \\
& \int_{\mathbb{R}} f(u) \frac{g(u-t)}{Q(u-t)} d u \int_{\mathbb{R}} \varphi(t) d t= \\
& \int_{\mathbb{R}}\left(f * \frac{g}{Q}\right)(t) \varphi(t) d t=<f * \frac{g}{Q}, \varphi>=<h, \varphi>.
\end{aligned}
$$

Note. In a similar way, it can be proven another version of this theorem. Namely, if $g \in L^{p}$ and if $f$ is measurable function such that $f / Q \in L^{1}$ then the convolution $(f / Q) * g \in L^{p}$ and, as before, it is proved that has Cauchy representation.

Theorem 5. Suppose that $f \in D^{\prime}{ }_{L^{p}}$ and $g \in D_{L^{q}}$, where $1 \leq p<$ $\infty, 1 \leq q<\infty$. Then the convolution of the distributions $f$ and $g, h=f * g$, has Cauchy representation.

Proof. For $f \in D^{\prime}{ }^{p}$ and $g \in D^{\prime}{ }_{L^{q}}$, we know that $h=f * g \in D^{\prime}{ }_{L^{r}}$, $\frac{1}{p}+\frac{1}{q}-1=\frac{1}{r}$.

By the structure theorem, we have that

$f=\sum_{i=1}^{n} f_{i}{ }^{(i)}$ and $g=\sum_{j=1}^{m} g_{j}{ }^{(j)}$, where $f_{i}{ }^{(i)} \in L^{p}$ and $g_{j}{ }^{(j)} \in L^{q}$.

Let $\varphi \in D$ be arbitrary function.

Then

$$
\begin{aligned}
& \lim _{y \rightarrow 0^{+}} \int_{\mathbb{R}}(\hat{h}(x+i y)-\hat{h}(x-i y)) \varphi(x) d x= \\
& \lim _{y \rightarrow 0^{+}} \int_{\mathbb{R}}\left(\frac{1}{2 \pi i} \int_{\mathbb{R}} \frac{h(t) d t}{t-z}-\frac{1}{2 \pi i} \int_{\mathbb{R}} \frac{h(t) d t}{t-\bar{z}}\right) \varphi(x) d x= \\
& \lim _{y \rightarrow 0^{+}} \int_{\mathbb{R}} \frac{y}{\pi} \int_{\mathbb{R}} \sum_{i=1}^{n} \sum_{j=1}^{m} f_{i}^{(i)}(u) g_{j}^{(j)}(t-u) d t d u \int_{\mathbb{R}} \frac{d t}{|t-z|^{2}} \varphi(x) d x=
\end{aligned}
$$




$$
\lim _{y \rightarrow 0^{+}} \int_{\mathbb{R}} \sum_{i=1}^{n} \sum_{j=1}^{m} \int_{\mathbb{R}} f_{i}^{(i)}(u) g_{j}^{(j)}(t-u) d t d u \frac{y}{\pi} \int_{\mathbb{R}} \frac{d t}{|t-z|^{2}} \varphi(x) d x .
$$

Since the integrals exist, by Fubini's theorem, we may change the order of integration and get

$$
\begin{aligned}
& \lim _{y \rightarrow 0^{+}} \int_{\mathbb{R}}(\hat{h}(x+i y)-\hat{h}(x-i y)) \varphi(x) d x= \\
& \lim _{y \rightarrow 0^{+}} \int_{\mathbb{R}} \sum_{i=1}^{n} f_{i}^{(i)}(u) d u \int_{\mathbb{R}} \sum_{j=1}^{m} g_{j}^{(j)}(t-u) d t \frac{y}{\pi} \int_{\mathbb{R}} \frac{\varphi(x) d x}{|t-z|^{2}} .
\end{aligned}
$$

Using the Lebesgue dominated convergence theorem and the Lemma 5.4, we obtain that

$$
\begin{aligned}
& \lim _{y \rightarrow 0^{+}} \int_{\mathbb{R}}(\hat{h}(x+i y)-\hat{h}(x-i y)) \varphi(x) d x= \\
& \int_{\mathbb{R}} \sum_{i=1}^{n} f_{i}{ }^{(i)}(u) d u \int_{\mathbb{R}} \sum_{j=1}^{m} g_{j}^{(j)}(t-u) \varphi(t) d t= \\
& \sum_{i=1}^{n} \sum_{j=1}^{m} \int_{\mathbb{R}} f_{i}{ }^{(i)}(u) d u \int_{\mathbb{R}} g_{j}^{(j)}(t-u) \varphi(t) d t .
\end{aligned}
$$

One more application of Fubini's theorem gives that

$$
\begin{aligned}
& \lim _{y \rightarrow 0^{+}} \int_{\mathbb{R}}(\hat{h}(x+i y)-\hat{h}(x-i y)) \varphi(x) d x= \\
& \sum_{i=1}^{n} \sum_{j=1}^{m} \int_{\mathbb{R}} f_{i}^{(i)}(u) g_{j}^{(j)}(t-u) d u \int_{\mathbb{R}} \varphi(t) d t= \\
& \sum_{i=1}^{n} \sum_{j=1}^{m} \int_{\mathbb{R}}\left(f_{i}^{(i)} * g_{j}^{(j)}\right)(t) \varphi(t) d t= \\
& \int_{\mathbb{R}} h(t) \varphi(t) d t=\langle h, \varphi\rangle .
\end{aligned}
$$




\section{REFERENCES}

[1] H. Bremerman, Raspredelenija, kompleksnije permenenije i preobrazovanija Furje, Izdatelstvo "Mir" Moskva, 1968.

[2] R. Carmichael, D. Mitrovic, Distributions and analytic functions, New York, 1989.

[3] V. Manova-Erakovic, V. Reckoski, A note on the analytic representations of convergent sequences in $S$ ', Filomat 29:6 (2015), University of Nis, Serbia, 2015, p.p. 1419-1424.

[4] V. Manova Erakovik, S. Pilipovic, V. Reckovski, Analytic representations of sequences in $L^{p}$ spaces, $1 \leq p<\infty$, Filomat 31:7 (2017), University of Nis, Serbia, 2017, p.p. 1959-1966.

[5] J. Pandey, The Hilbert transform of Schwartz distributions and applications, John Wiley \& Sons, Inc, 1996.

[6] W. Rudin, Functional Analysis, Mc Graw-Hill, Inc., 1970.

FACUlty of TOURISM AND Hospitality, UniVERsity St. KLIMENT OHRIDSKI,

Bitola, Republic of Macedonia

E-mail address: vaskorecko@yahoo.com

University Mother Teresa,

FACULTY OF INFORMATICS,

Ul. 12 Udarna Brigada, Br. 2A, Kat 7, 1000, Skopje, Republic of Macedonia

E-mail address: egzona.iseni@unt.edu.mk

Ss. Cyril And Methodius University,

Faculty of Mathematics and Natural Sciences,

Arhimedova bB, Gazi baba, 1000, Skopje, Republic of Macedonia

E-mail address: vesname@pmf.ukim.mk 